\title{
Primary Frequency Regulation Based on Deloaded Control, ANN, and 3D-Fuzzy Logic Controller for Hybrid Autonomous Microgrid
}

\author{
Assia Mahrouch $^{1}$ [ $\cdot$ Mohammed Ouassaid ${ }^{1}$
}

Received: 7 October 2020 / Accepted: 19 January 2022 / Published online: 26 January 2022

(c) The Author(s), under exclusive licence to Springer Nature Singapore Pte Ltd. 2022

\begin{abstract}
This study proposes an original approach to improve the Primary Frequency Regulation (PFR) of an Autonomous Microgrid $(\mathrm{A}-\mu \mathrm{G})$. The Wind Turbine Generator (WTG) is considered to be a principal resource. Due to the wind variability and intermittency, Diesel Generator (DG) is incorporated to meet the load peak. However, to reduce the system maintenance and fuel consumption and to increase the microgrid efficiency, Battery Energy Storage System (BESS) is added. Hence, to meet the load demand, the A- $\mu \mathrm{G}$ relies on the strengths of each technology. Among these various system components, an Intelligent Energy Management System (IEMS) is built in two stages to manage the $\mu \mathrm{G}$. First, the deloaded method is adopted to use the rotational kinetic energy, as a reserve, when the wind speed is low or the power consumption is high. Second, aiming at enhancing the dynamic of deloaded WTG and the DG participation, two intelligent-based control strategies, Artificial Neural Network (ANN) and three-dimensional Fuzzy Logic-Frequency Regulation (3D-FL-FR), are designed and compared. Different case studies and circumstances have been performed to test the efficiency of the adopted IEMS. The performances of both approaches have been proven in terms of guaranteeing the power balance of the A- $\mu \mathrm{G}$ and improving the primary frequency regulation. Indeed, with 3D-FL-FR combined with the DC, the frequency deviation is $-2.8 \%$, while the ANN control with DC records a frequency deviation of $-3.6 \%$. Therefore, both intelligent based controllers comply with the IEEE Std 1547-2003, even under large load demand and wind power fluctuation. However, the comparison results reveal the supremacy of the 3D-FL-FR against the ANN-based IEMS.
\end{abstract}

Keywords Autonomous microgrid · Fuzzy logic control · Artificial neural network · Battery energy storage system · Deloaded control · Energy management

\section{Introduction}

\section{Context and Related Works}

Resources based on Renewable Energy are growing over the world. They are the future of the power systems, as well as they are inexhaustible and protect the environment [1]. Hence, they growth as a potential solution to electrifying off-grid communities. Indeed, according to the International Energy Agency (IEA), in Africa, the onshore wind

Assia Mahrouch

assiamahrouch@ research.emi.ac.ma

Mohammed Ouassaid

ouassaid@emi.ac.ma

$1 \quad$ Engineering for smart and sustainable systems research center, Mohammadia School of Engineers, Mohammed V University, Rabat, Morocco generation will reach 2000 TWh by 2022. The recent reports of the IEA have shown that the total electrification percentage is $89 \%$ and 153 million people use electricity every year. However, the main defiance is that in the most remote areas, globally and $95 \%$ of those living without electricity are in countries in Sub-Saharan Africa where 573 million people still in the darkness. Consequently, as the most economical, suitable, and efficient systems, $\mathrm{A}-\mu \mathrm{G}$ scenarios are taken into account [2]. The $\mu \mathrm{G}$ is a power system capable to solve the electrification problem in remote areas, which comprises one or more renewable sources (wind, PV, Hydraulic, etc.) and one or more conventional sources with an energy storage system [3-5].

The reliable power supply to the consumer under varying weather conditions is one of the major $\mu \mathrm{G}$ aspects based on wind energy. To cope with this issue, the integration of a Diesel Generator (DG) and Batteries is a solution. In the literature, reasonable research is done on DG as a synchronous 
generator to satisfy the load demand in hard cases [6]. However, knowing that the DG performs effectively when it is functioning around its rated capacity, huge changes in the load demand may cause poor power quality performances. In addition, the DG fuel cost is expensive and varies over the year [7]. Therefore, to guarantee higher efficiency and to reduce diesel consumption, researchers generally agree that the incorporation of the BESS with the wind generator and DG is a reliable and cost-effective option $[8,9]$. In this configuration, batteries play an important role. Indeed, batteries not only contribute to lessen the wind power oscillations, increase the system reliability and relief the DG during low load demand, but also provide ancillary services to the A- $\mu \mathrm{G}[10]$. Hence, augmenting the $\mu \mathrm{G}$ with batteries can be an effective way to improve system frequency response. With strong advantages like controllability, the response at a short time, which can adjust the unexpected frequency fluctuation, and increasing system accuracy, the battery has attracted considerable attention [11]. Energy storing by battery are gaining more and more attention and used as an effective solution [12]. There are varieties of batteries to store energy; however, the Lead-acid Batteries (LABs) are the most attractive in this case. They are recyclable and present a safe, cost-effective solution, which can be adapted for different energy storage applications types [13], and it is suitable for storing energy for long-time periods. Furthermore, the LAB is among the most demanded batteries in the renewable energy market due to its power density, efficiency, and the best load shifting performance [14]. They are available in various sizes and designs [15].

Many research studies proceeded to deal with modeling, optimization, and management of $\mathrm{A}-\mu \mathrm{G}$, but few of them are tackling the stability and frequency problem controls. The objective of this study is to improve the performance and the capability of an A- $\mu \mathrm{G}$ composed of a Permanent Magnet Synchronous Generator (PMSG)-based Wind Turbines (WTs), DG, batteries, and residential load. The frequency control problem of the $\mu \mathrm{G}$, under the nominal operating condition, mainly focuses on load perturbations.

Because of the intermittent wind velocity and the load demand, IEMS is designed to supervise the power flow among the different components of the A- $\mu \mathrm{G}$. To extract the maximum wind power, while ensuring a power reserve to handle the frequency problem control, a deloaded control and MPPT control are adopted. Moreover, The IEMS is optimized to power the load and to keep the battery SOC between certain target margins.

The proposed strategy is based on artificial intelligence techniques, including 3D-FL-FR and ANN. Indeed, during the last two decades, intelligent modeling methods based on ANN and Fuzzy Logic Control (FLC) have become popular and have been used by many researchers for a variety of engineering applications $[16,17]$. On the one hand, FLC systems are resilient device based on artificial intelligence [18]. On the other hand, ANN is based on human brain, has proven to be well suited for IEMS control, by decreasing the level of errors, and is capable to outperform the previously developed techniques [19].

\section{Main Contributions}

As aforementioned, the added value of this study is summarized as follows:

- Incorporation of BESS to enhance the overall performance of autonomous microgrid based on diesel and wind systems.

- Preserving the operating frequency in a normal range, by eliminating the negative effect of both load demand variation and wind intermittency.

- Developing a comprehensive IEMS based on 3D-FL-FR and ANN to support primary frequency control via MPPT and DC.

- The IEMS is designed to satisfy the load demand while reducing the diesel consumption and controlling the SOC to avoid damage to the battery.

\section{Description of the Studied System and Mathematical Model}

\section{The Studied Microgrid System Representation}

The proposed $\mu \mathrm{G}$ consists of wind farm (WF), DG, batteries, and load managed using IEMS as can be seen in Fig. 1. They are coupled to the generation bus by converters. In this typology, wind energy is designed to be the principal source of power generation. The WF is based on PMSGs thanks to its attractive features such as high reliability and efficiency, self-excitation, brushless design, gear-less, high accuracy, adequate execution [20-22], MPPT capability, low noise emissions $[23,24]$. The WF, DG, and BESS are coordinately functioning in order to respond to the load consumption together. Indeed, the DG is implemented as a relief to reduce the fluctuation nature of the wind generator and to control the system frequency $[25,26]$. Additionally, DG is integrated to avoid blackouts. Batteries, as emergency elements, can charge fast and discharge slowly, when is necessary. It is controlled to suppress unexpected error fluctuation [27], and to support primary frequency in the $\mu \mathrm{G}[28-32]$.

The load demand changes constantly in the function of time, which leads to an imbalance between active power supply and demand. The power margin is calculated using (1):

$\Delta P=P_{D G}+P_{W F} \pm P_{B E S S}-P_{L}$ 
Fig. 1 The proposed $A-\mu \mathrm{G}$ scheme

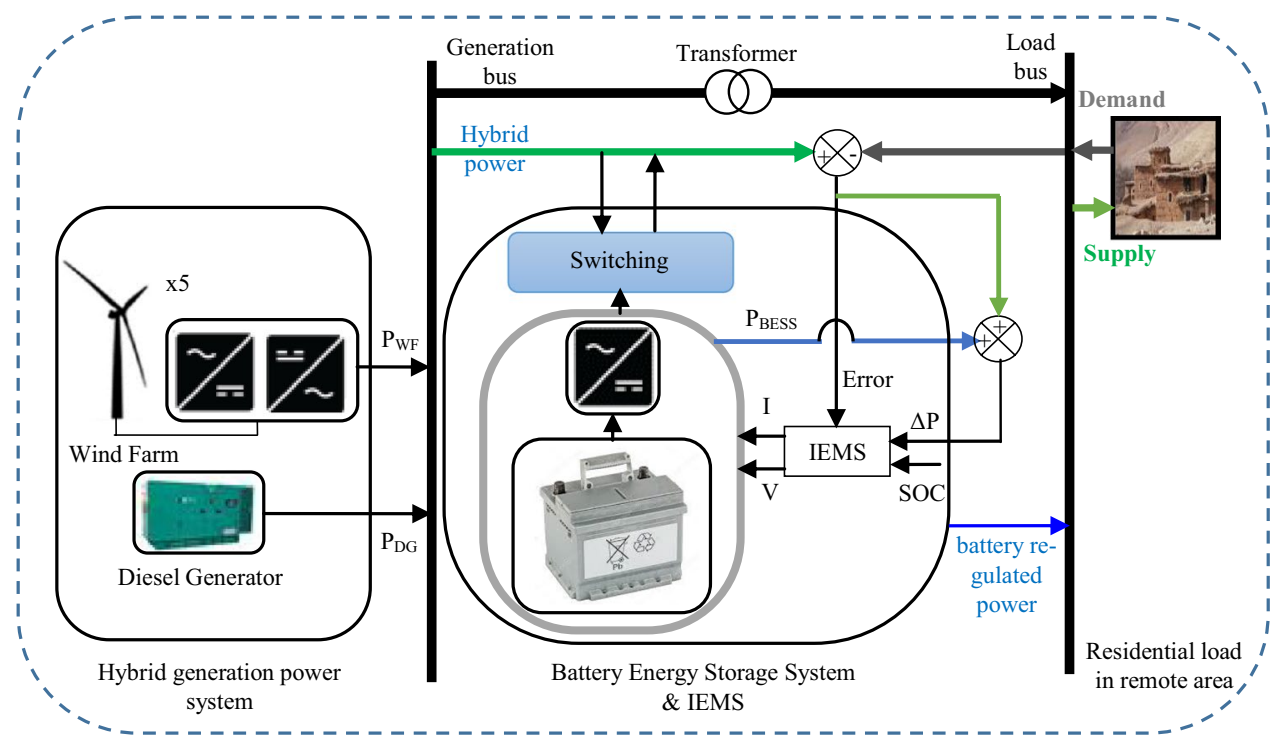

where $P_{D G}, P_{W F}, P_{B E S S}$, and $P_{L}$ are the DG output power, WF power, battery power, and load demand power, respectively.

One of the key roles of the proposed IEMS is to maintain the power deviation, which reflects the frequency fluctuation, converging to zero, whatever the disturbance and its source. Thus, the proposed IEMS is designed to control the pitch angle in the presented power system using the DC technique based on 3D-FL-FR and ANN. The studied system can automatically switch following the subsequent conditions:

(i) If the power generated by the hybrid system (wind generator and DG exceeds the power required, from the load), the surplus is stored in the battery bank.

(ii) When the power required is greater than the power generated, the battery bank partakes to assure the load demand.

\section{Deloaded and MPPT Operating Modes of WTG}

The PMSG is driven by the WT and linked to the A- $\mu \mathrm{G}$ via a back-to-back converter, as depicted in Fig. 1. The extracted power $P_{m}$ is expressed as follows:

$P_{m}=0.5 \rho \cdot S \cdot C p(\lambda, \beta) \cdot v^{3}$

where $\rho$ presents the density of air $\left(\mathrm{kg} \cdot \mathrm{m}^{-3}\right), S$ is the area swiped by the WT blades $\left(\mathrm{m}^{2}\right), v$ presents the wind speed (m.s ${ }^{-1}$ ) and $C_{p}$ presents the power constant given in (3):

$C_{p}(\lambda, \beta)=0.5\left[\left(\frac{116}{\lambda_{i}}\right)-0.4 \beta-5\right] e^{\left(\frac{-21}{\lambda_{i}}\right)}$ with

$\frac{1}{\lambda_{i}}=\frac{1}{\lambda+0.08 \beta}-\frac{0.035}{\beta^{3}+1}$

In the studied WTG, the value of $C_{p}$ is 0.41 reached when the optimal ratio of the tip-speed $\lambda_{\text {opt }}=8.1$ and pitch angle $\beta=0^{\circ}$. The description of any size of WT is given by $C_{p}$ and $\lambda$. wherever, $\lambda$ is given by (5):

$\lambda=\frac{R \cdot \omega_{r}}{v}$

where $R$ presents the blade radius of the WT (m) and $\omega_{r}$ is the WT rotor angular speed $\left(\operatorname{rad} . \mathrm{s}^{-1}\right)$. Using (2) and (5), the mechanical torque (aerodynamic torque), in terms of the mechanical power $\left(P_{m}\right)$, can be computed as follow:

$T_{m}=\frac{P_{m}}{\omega_{r}}=0.5 \rho \cdot S \cdot R \cdot \frac{C p(\lambda, \beta)}{\lambda} \cdot v^{2}$

In MPPT mode, the WTGs operate so that they capture the maximum power by monitoring the rotor speed or the pitch angle. Consequently, the operating point is at the maximum $C_{p}$ curve, as can be noticed in Fig. 2. When it comes to contributing to the PFR, it is required that WTGs have a $10 \%$ power reserve. Therefore, they operate at reduced power corresponding to the deloaded curve $P_{d}\left(\omega_{r . d e l}\right)$ from MPPT curve $P_{m}\left(\omega_{\max }\right)$. The delaoded control allows the PMSG to excerpt additional kinetic energy, which can be used as stored energy to enhance the frequency stability and help in enhancing the quality of the generated power during large load change [33]. The deloaded and MPPT curves of the rotor speed are given 


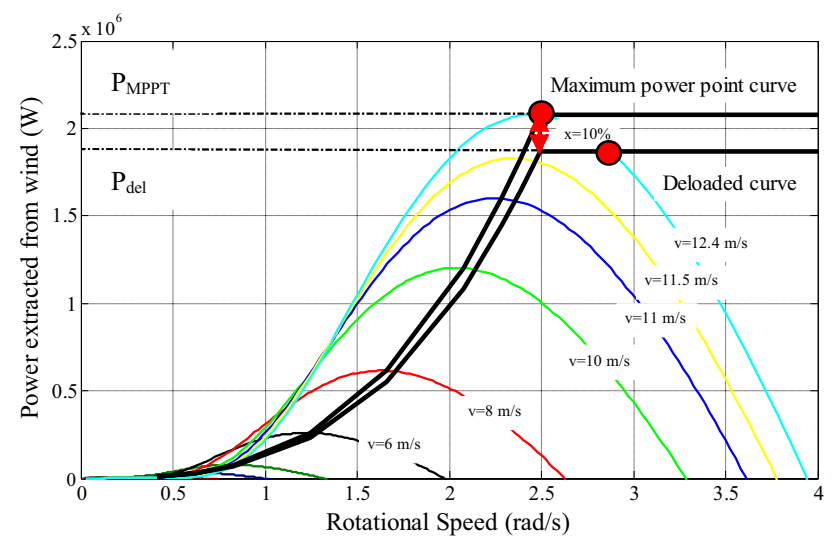

Fig. 2 Deloaded and MPPT curves versus wind speed

in Fig. 2. Wherever, WTGs can function in both, underspeeding (the left side) and over-speeding (the right side) of the power extracted from the wind. Typically, WTGs based-deloaded control, work on the curve on the right side to enhance system security [34].

Authors in [34] discussed $\left(P_{\text {ref }}\right)$ a relation linking the referenced deloaded power and the speed of the WT rotor. The $P_{\text {ref }}$ in terms of rotor velocity, power reserve $\left(P_{\text {reserve }}\right)$, and deloaded power $\left(P_{d e l}\right)$ is calculated as follows:

$P_{r e f}=P_{d e l}+\mathrm{P}_{\mathrm{resrve}}\left[\frac{\omega_{r, d}-\omega_{r}}{\omega_{r, d}-\omega_{r, o p t}}\right]$

where $\omega_{r, d}$ is the angular speed of the rotor at $P_{d e l}, \omega_{r, o p t}$ is the angular speed of the rotor at MPPT. $P_{d e l}$ is related to the maximum extracted power $\left(P_{M P P T}\right)$ by the mean of the required power margin $(x) . P_{d e l}$ can be calculated as follows:

$P_{d e l}=P_{\mathrm{MPPT}}(1-x)$

where $x$ presents the reserve power (around 10\%) reserved in the rotating mass in form of kinetic energy.

\section{Diesel Generator Modeling}

The wind is the main power resource the $\mathrm{A}-\mu \mathrm{G}$. Knowing that WTG power is relative to the wind velocity cubic power. Therefore, a lesser fluctuation in wind velocity may result in instability of the A- $\mu \mathrm{G}$. To meet this challenge, a DG is commonly used as a backup (Fig. 1), and it has led to A- $\mu \mathrm{G}$ as an attractive solution for remote area power supply $[35,36]$.

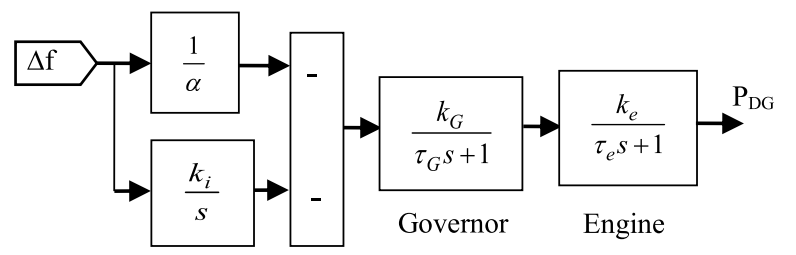

Fig. 3 Diesel generator modeling

The DGs are modeled taking into account their consumption of fuel and their efficiency [2]. The DG fuel consumption is expressed using (9):

$F(t)=0.246 P_{D G}(t)+0.08415 P_{r}$

where $F(t), P_{D G}$, and $P_{r}$ present the consumption of fuel $\left(\right.$ L.hour $\left.^{-1}\right)$, the generated power $(\mathrm{kW})$, and the DG rated power $(\mathrm{kW})$, respectively.

In the same way, the efficiency of DG is determined as follows:

$\eta_{\text {total }}=\eta_{\text {brake-thermal }} \times \eta_{\text {generator }}$

where $\eta_{\text {total }}$ presents the DG total efficiency (\%), $\eta_{\text {brake-thermal }}$ presents the DG brake-thermal efficiency (\%), and $\eta_{\text {generator }}$ presents the generator efficiency (\%).

Figure 3 shows the mathematical model adopted in this study [37].

\section{Lead Acid Battery Modeling}

The DGs are characterized by their large delays. Hence, their response to the frequency fluctuations may not be quick enough. Therefore, to overcome this drawback, energy buffers are integrated to positively influence the frequency regulation.

To cope with the renewable energy intermittency, LABs are the preferred kind of battery, because they are not heavy in transportation, recyclable, and comparatively lower price compared to other types [38, 39]. In general, according to [39], ohm's law describes the battery behavior:

$V=I, R_{i}$

where the current $I$ is the current density $\left(\mathrm{A} / \mathrm{cm}^{2}\right)$ and $R_{i}$ is the internal resistance.

The following equation presents the charging equation:

$V_{c h}=\left[2-\frac{16}{100} \times S O C\right]-\frac{I}{C_{n}} \times\left(\frac{6}{1+I^{0.86}}+\frac{0.48}{(1-S O C)^{1.2}}+\frac{36}{1000}\right) \times\left(1-\frac{25}{1000} \times \Delta T\right)$ 
where $C_{n}$ is the rated capacity of the LAB. The following exponential function represents the overcharge phenomenon (gassing):

$V_{\text {over-ch }}=V_{g}+\left[1-\exp \left(\frac{0.95 C-A h_{\text {restored }}}{I . \tau}\right)\right] \times\left(V_{e c}-V_{g}\right)$

where $V_{g}$ is the voltage of gassing; $V_{e c}$ is the voltages of the final charge $(\mathrm{V})$; with regard to the battery capacity $C$, $A h_{\text {restored }}$ characterizes the ampere-hours stored in the battery; and as an estimation, $\tau$ (the time constant), can be given as:

$$
\tau=\frac{17.3}{1+852\left(\frac{I}{C}\right)^{1.67}}
$$

In addition, according to [38], the battery discharging voltage evolves as follows (15): different technical constraints. Frequency deviation is the indicator of the active power mismatches. Figure 4 exhibits the overall organization of the adopted IEMS and Fig. 5 presents the energy management flowchart.

The primary frequency response is provided by the proposed IEMS using BESS as fast responsive energy storage technology. Wherever the incremental change in frequency $(\Delta f)$ and power $(\Delta P)$ is related as follows $[33,34]$ :

$\Delta f=-\alpha \Delta P$

where $\alpha$ is the coefficient constant of the frequency droop. The drop may vary from $3 \%$ to $6 \%$, according to each country grid code, for all units taking part within the system frequency regulation.

The BESS participation in primary frequency response is shown in Fig. 6. If the frequency value exceeds $50 \mathrm{~Hz}$

$V_{\text {disch }}=\left[\frac{1965}{1000}+\frac{12}{100} \times S O C\right]-\frac{I}{C_{n}} \times\left(\frac{4}{1+I^{1.3}}+\frac{0.27}{S O C^{1.5}}+\frac{2}{100}\right) \times\left(1-\frac{7}{1000} \Delta T\right)$

where (15) represents the variation of the open circuit voltage, using SOC, and the variation of the internal resistance, calculated by the series resistance sum. Moreover, (16) gives the temperature variation of the battery $(\Delta T)$ :

$\Delta T=T-25^{\circ}$

The relationship between SOC and Depth of Discharge (DOD) is calculated as follows:

$$
S O C=1-D O D
$$

\section{The Proposed Intelligent Energy Management System}

To monitor the mode operation and the power flow, a supervisory control unit, IEMS, is built. Consequently, the IEMS is the $\mathrm{A}-\mu \mathrm{G}$ heart, which ensures the balance between the power generation and the load demand, while handling (over-frequency) or is inferior to $50 \mathrm{~Hz}$ (under-frequency), the battery is responsible for the inertial response of conventional and wind turbine generators by absorbing/liberating the active power from/to the $\mathrm{A}-\mu \mathrm{G}$. To enhance the IEMS, the pitch angle is controlled using 3D-FL-FR control, then ANN.

Figure 7 depicts the block diagram of the proposed technique. Controlling the pitch angle is powerful and extensively used to adjust the WT aerodynamic torque when the wind speed exceeds the estimated speed. Indeed, the exploitation of the created power reserve can be varied between $P_{d}$ and $P_{m}$. This is allowed through varying the rotor speed between $\omega_{r . d e l}$ and $\omega_{r \text { max }}$, as depicted in Fig. 2 . Pitch controller dynamics are realized using two intelligent based strategies, along with a servomechanism to stabilize its response. Therefore, the over-speeding technique is used in the IEMS corresponding to low, medium, and high wind speed to enhance DC and to obtain the desired $P_{\text {out }}$.
Fig. 4 Block diagram 3D-FLFR and ANN for the PFR

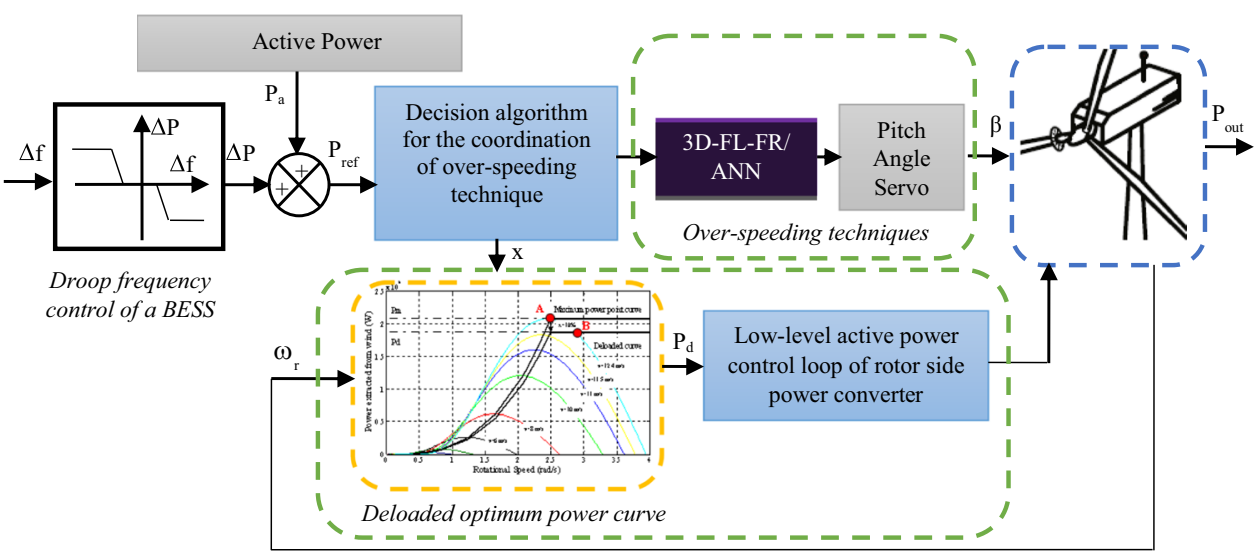


Fig. 5 Energy management flowchart

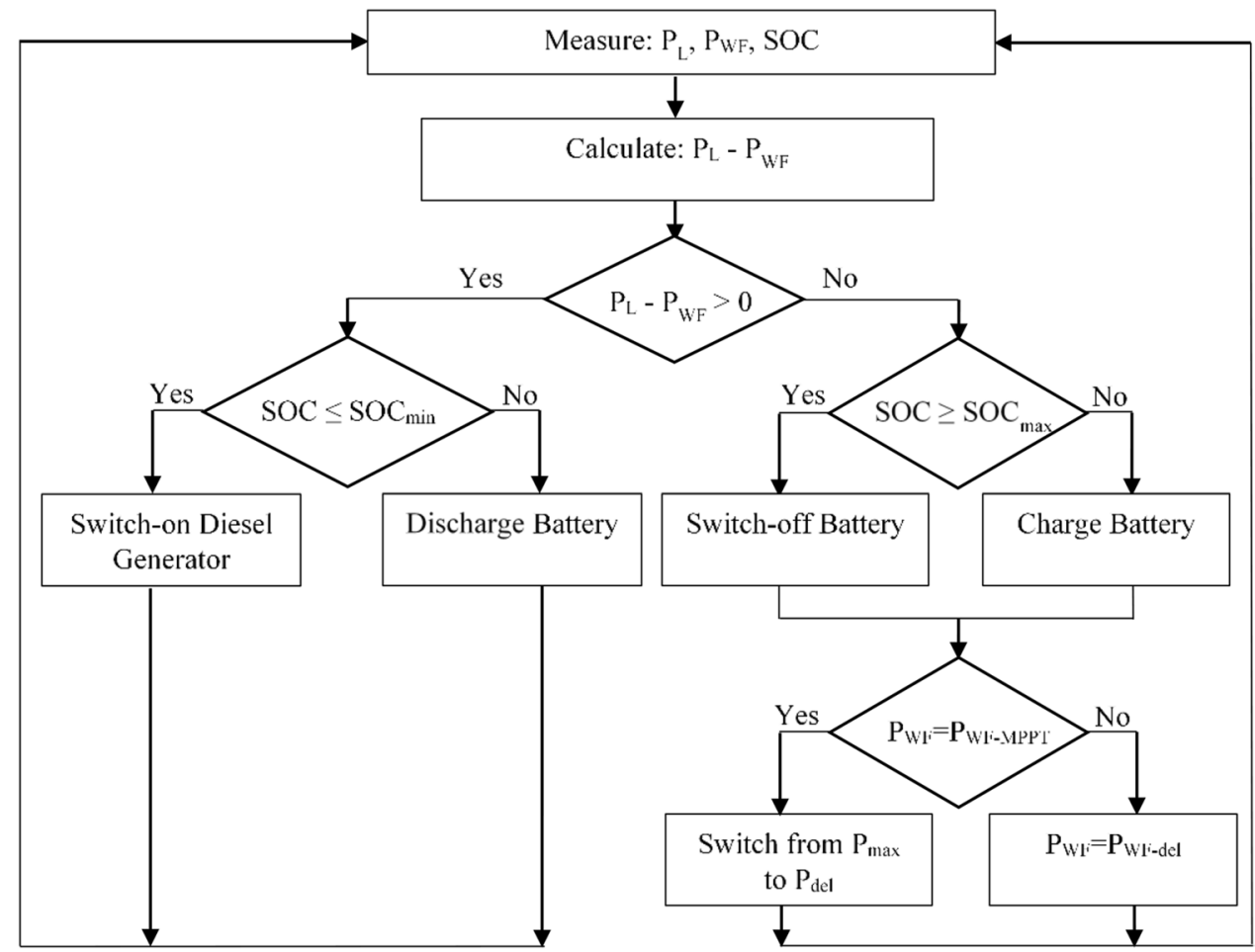

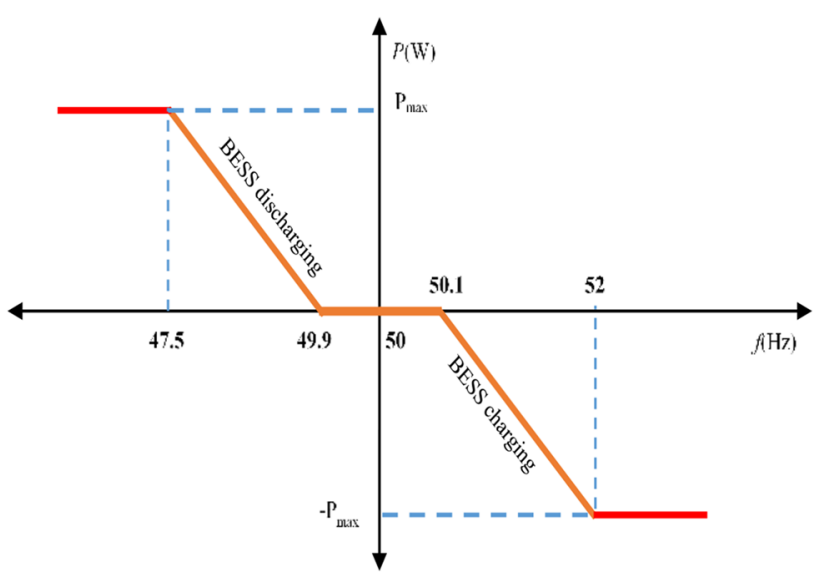

Fig. 6 BESS participation in primary frequency response

In the following section, two intelligent controllers are developed, and then their results are compared. The first one is based on fuzzy logic, whereas the second one is based on ANN.

\section{The Three-Dimensional Fuzzy-Logic Frequency Regulatio}

With the aim to compensate for the change of the primary frequency, the desired $P_{\text {out }}$ is calculated through the pitch angle, which is determined using frequency deviation $(\Delta f)$ and rotor speed $\left(\omega_{r}\right)$.
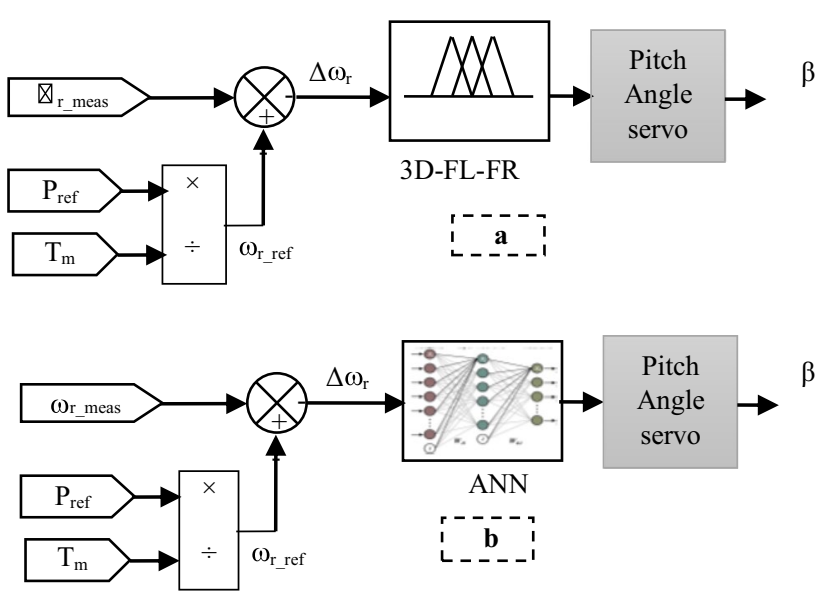

Fig. 7 Modeling of the proposed over-speed technique with 3D-FLFR (a) and ANN (b)

Figure 7.a and Fig. 8 show the block diagram and the $3 \mathrm{D}$ representation of the proposed 3D-FL-FR. The inputs of the 3D-FL-FR are " $\Delta f$ " and " $\omega_{r}$ " and output is " $\beta$ ". The fuzzy range variables taken in this paper are $\Delta \mathrm{f}=[-0.4: 0.1: 0] \mathrm{Hz}$, and $\omega_{\mathrm{r}}=[2.4: 0.1: 2.6] \mathrm{rad} / \mathrm{s}$. The output is "pitch angle" to control the generated wind power during high wind speeds and deloaded operation. The general structure of FLC, which consists of three steps named Fuzzification, fuzzy logic rules, and defuzzification [40], is adopted in this work. To convert the inputs into appropriate linguistic values, the use of Fuzzification is 


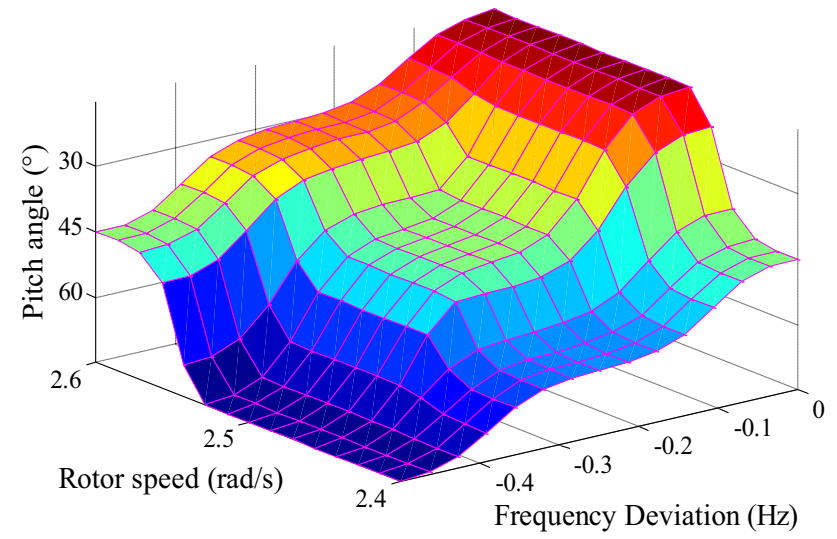

Fig. 8 The frequency deviation and rotor speed variation influence on the pitch angle via three-dimensional FL-FR

necessary. The Fuzzy rule base covers rules that comprise all possible fuzzy relations between inputs and output. Finally, defuzzification presents the alteration of the output language, which is transformed into an existent value using the gravity method center.

The used membership functions are described as follows: Negative Big (NB), Negative Medium (NM), Negative Small (NS), Zero (ZO), Positive Small (PS), Positive Medium (PM), Positive Big (PB). The Gaussian membership illustrates these membership functions.

Fuzzy logic rules draw the positioning of 3D-FL-FR, as shown in Table 1 and Fig. 9. They are defined by the "IFTHEN" rules set. Therefore, IF the tracking control error $\left(\Delta \omega_{r}\right)$ between $\omega_{r-\text { ref }}$ and $\omega_{r-\text { meas }}$ is nonzero, THEN the variation of the proposed control should be consequently modified to refer to the control error state of zero. Furthermore, it is also relatively evident that IF $\Delta \omega_{r}$ is nonzero AND the initial period derived of the discovering control role is negatively small (decreasing) THEN the variety of the control has to be shorter with respecting the previous case. As it can be noticed, the developed procedure is capable to automatically construct the fuzzy rules.

\section{The Proposed Artificial Neural Network Controller}

In this Work, Sigmoid ANN Is Used as a Smart Mathematical Process, Implies Three Principal Parts: Input, Hidden, and Output Layer. The Input Layer where the First Data
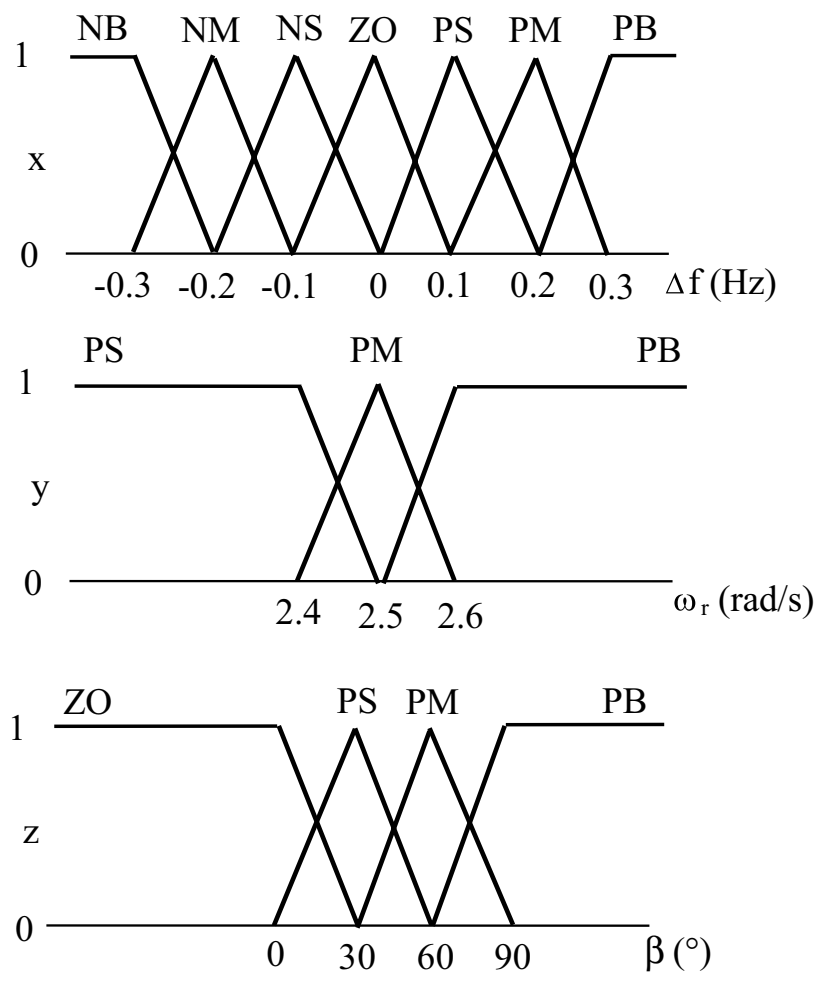

Fig. 9 Fuzzy Logic proposition Membership functions

for the ANN Are Communicated; the Intermediary Layer in the Midst of the Input and Output Layer, the Position where all the Calculations Are Done, Called Hidden Layers Are. Every Neuron in the Hidden Level Is Connected to the Totality Neurons in the Output Level. From the Last One, the Performances of the Conversion Are Obtained. The Received and Sent Values Depending on the Weight of the Channel Significance that Transmits the Value to/from the Neuron. The Weight of the Channel Means the Multiplied Value by the Approved Estimation Crossing Previously the Result to the Next Neuron. The Weighty Significance Varies with Varying the Proposed Task. Therefore, the Instructing Operation and Calculations in the Layers and Neurons Occur Using the Input-Output Equation Given as Follows

$$
y_{\text {out }}=\sum_{i=1}^{M} w_{i} x_{i}
$$

Table 1 Fuzzy logic rules

\begin{tabular}{llllllll}
\hline $\begin{array}{l}\Delta \mathrm{f} \\
\omega_{\mathrm{r}} / \Delta \omega_{\mathrm{r}}\end{array}$ & NB & NM & NS & ZO & PS & PM & PB \\
\hline PS & PB & PM & PM & PS & PS & ZO & ZO \\
PM & PB & PB & PM & PM & PB & PB & ZO \\
PB & PB & PB & PB & PM & PM & PS & PS \\
\hline
\end{tabular}


where $w_{i}$ are the weights for each input $x_{i}$ and $y_{\text {out }}$ is the output. Whereas, from [41], the sigmoid activation function is expressed as follows:

$\operatorname{sgm}(x)=\frac{1}{1+\exp (-x)}$

The real and imaginary parts of the variables $\Delta f$ and $\omega_{r}$ are separately fed to the sigmoid type ANN, bringing the relation $2 \times 3 \times 1$, two inputs, three hidden, and one output layer. Indeed, the adopted network structure is known as ANN 2-n-m-1, which contains $n$ nodes in the first hidden layer, $m$ nodes in the second hidden layer, one input node and two output nodes.

In the Same Context, an ANN-Based Controller of the Frequency Deviation Is Conceived in this Subsection. The Structure of the Adopted Controller Is Exhibited in Fig. 7.B. as Shown in Fig. 10, the ANN Controller Takes as Inputs Layers, $\Delta f$ and $\omega_{r}(\mathrm{~N}=2)$, the Number of Nodes in the Second Hidden Layer Is Three $(\mathrm{M}=3)$, and One Output Layer, which Is the Pitch Angle to Regulate $P_{\text {out }}$ for the Period of High Wind Speeds and Deloaded Operation

\section{Simulation Results and Discussion}

To validate the efficacy of the two adopted 3D-FL-FR and ANN controllers based IEMS, the architecture of the A- $\mu \mathrm{G}$, which is depicted in Fig. 1, is built in the MATLAB/Simulink environment. The proposed microgrid is composed of five WTG-based PMSGs with 2 MW of capacity for each one, one DG, and twelve LABs with more than eight hours of autonomy. The parameters of all used assets are shown in Table 3.

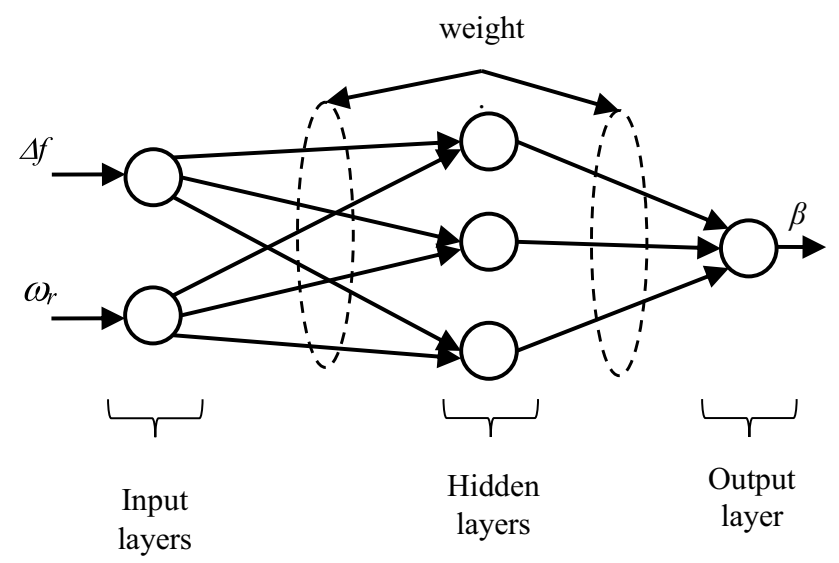

Fig. 10 Structure of the proposed sigmoid ANN
The IEMS is designed to permit the algorithm to cope with frequent variation in the system, observe and respond to the small changes in the load and generation.

For comparison purposes, different configurations of the IEMS, WTG with and without DC, pitch angle with 3D-FLFR, or ANN has been investigated. Moreover, various scenarios of wind power availability and load demand variation have been considered.

Figure 11.a illustrates the load demand variation $(+1.9 \mathrm{MW}$ at $140 \mathrm{~s}, 4.2 \mathrm{MW}$ at $270 \mathrm{~s}$, and $-0.2 \mathrm{MW}$ at 370 s), while Fig. 11.b shows a model of wind speed fluctuations between approximately 7 and $11 \mathrm{~m} / \mathrm{s}$.

\section{A. ANN controller-based IEMS controller performance}

\section{Case a.1: Wind Fluctuation Response with and without DC}

As it can be noticed in Fig. 11.c, during the first stage, from 0 to $140 \mathrm{~s}$, the load demand is constant and the frequency deviation equals zero, despite the wind fluctuations. When a load variation occurs, with the proposed control without DC, the frequency drop lasts $6 \mathrm{~s}$ (between $270 \mathrm{~s}$ and $276 \mathrm{~s}$ ) to be stabilized, which explains the effectiveness of DC in primary frequency regulation. On the other hand, Fig. 11.d shows that active power follows wind speed variations when the DC mode is not active. A rate of $10 \%$ is the desired power margin between MPPT and DC curves as studied at [33, 34], this percentage represents the power reserved by WT using deloaded control approach. On the contrary, when the DC mode is switched on, the out power is smoothed, thanks to the association pitch angle and rotor speed controllers and the participation of BESS and DG, as can be noticed in Fig. 12 and Fig. 13.

\section{Case a.2: Response to Step Load Change with and with- out DC}

When load changes suddenly, a variation of $4.2 \mathrm{MW}$ at 270 s, as illustrated in Fig. 11.a, Fig. 11.c demonstrates the frequency deviation $\Delta f$ is about $-6.7 \mathrm{~Hz}$ without deloaded control but decreases to $-1.8 \mathrm{~Hz}$ when the deloaded control is activated. This is due to the fact the system is capable to deliver a reserve power of $2.7 \mathrm{MW}$, due to auto-storage offered by deloaded control.

The DG share presents $21 \%$ of the total supplied power to minimize the need to fuel at the same time and to satisfy the load demand if needed. It is worth noticing that DG participates in the supplied power, as shown in Fig. 12.b. However, the delivered power is lower under DC mode, which 

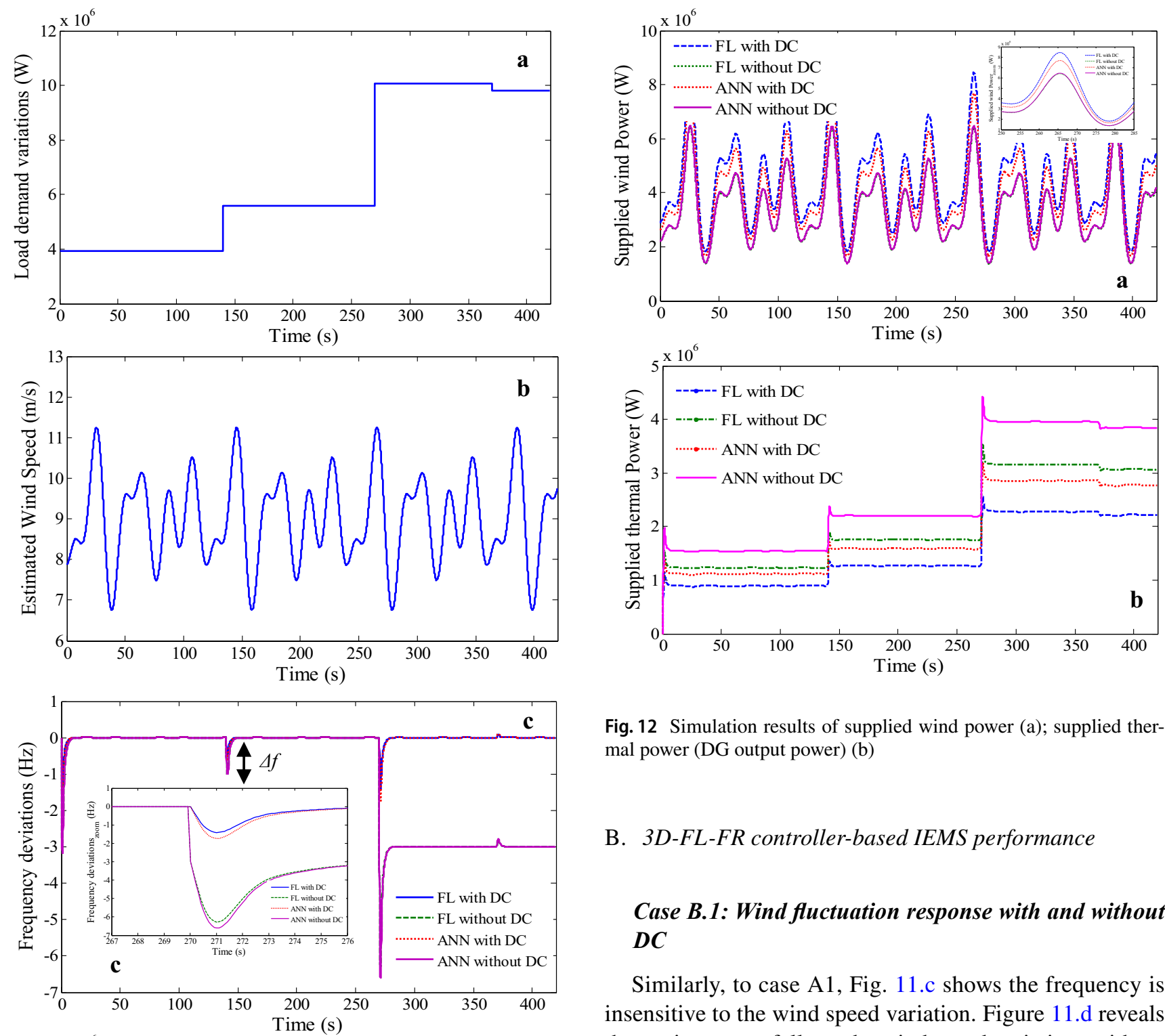

Fig. 12 Simulation results of supplied wind power (a); supplied thermal power (DG output power) (b)

\section{B. 3D-FL-FR controller-based IEMS performance}

\section{Case B.1: Wind fluctuation response with and without DC}

Similarly, to case A1, Fig. 11.c shows the frequency is insensitive to the wind speed variation. Figure 11.d reveals that active power follows the wind speed variations without DC. Referring to the same figure, the DC is efficient over the attenuation of the WTG power fluctuation.

\section{Case B.2: Response to Step Load Change with and with- out DC}

The frequency deviates under load power variation, as it can be observed in Fig. 11.c. However, it is easy to notice that with DC, $\Delta f$ is more mitigated with 3D-FL-FR and the maximum deviation is kept on $-1.4 \mathrm{~Hz}(-2.8 \%)$, which meets the tight frequency band $( \pm 5 \%)$ recommended by IEEE Std 1547-2003 [42], instead of -6 Hz recorded with-

Fig. 11 Simulation results of Load Power Demand (a); wind speed (b); frequency variations (c); active power (d) out DC. This result is obtained in spite of large wind speed perturbation.

Figure 11.d shows that the request of the load is mostly satisfied by the supplied wind power while the DC mode operates to continuously adjust the needed power. At the same

permits reducing fuel consumption. The positive effect of DC is obvious, as can be noticed in Fig. 12.b. 

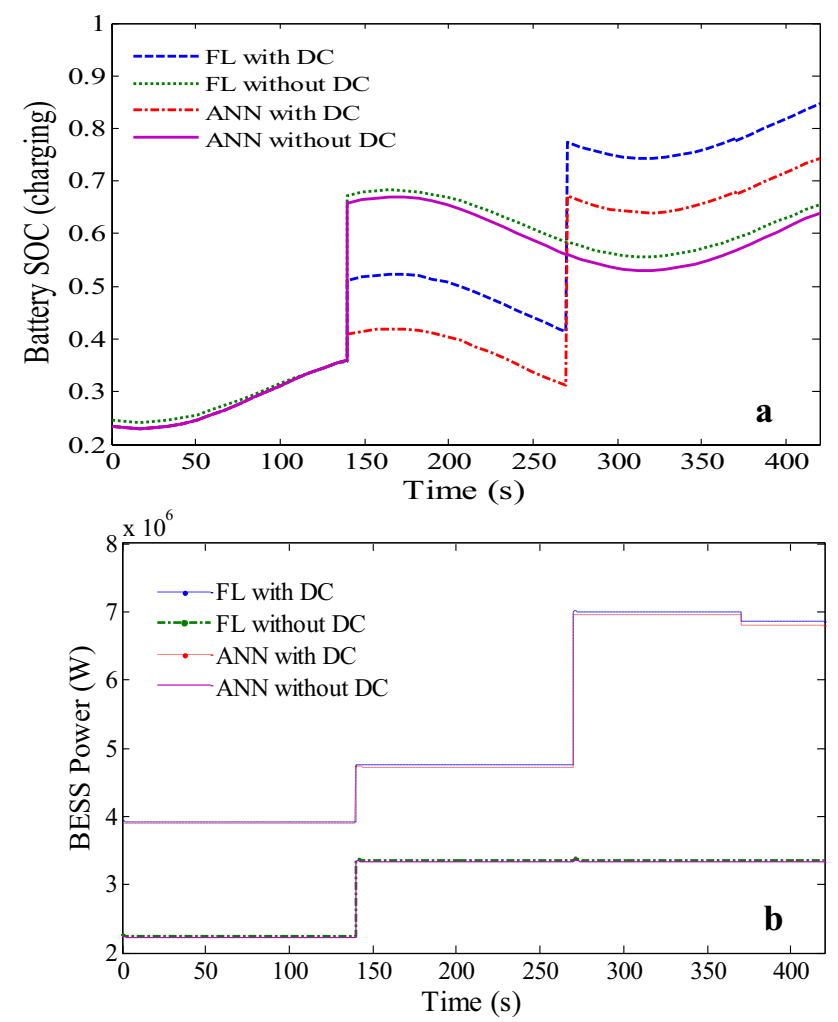

Fig. 13 Simulation results of battery stat of charge; battery supplied power (b) when the wind farm work

time, the IEMS maintains the output power at deloaded WTG mode, in order to establish the balance, case of large and sudden variation occurs. The obtained results prove the supremacy of the 3D-FL-FR controller against the ANN controller.

Again, the positive effect of the DC, regarding the amount of the thermal power converted, is very clear, as it can be comprehended from Fig. 12.b. Moreover, with the fuzzybased dynamic deloading control, the DG is less solicited to guarantee frequency stability.

Referring to Fig. 13.b, the simulation results approve the role of the BESS. Indeed, under the DC the LAB is rapidly and effectively contributing to meet the sudden and large variety of load demand. As a result, diesel consumption is noticeably reduced, leading to a decrease in $\mathrm{CO}_{2}$ emission. In addition, as can be observed in Fig. 13.a the IEMS succeeds to maintain the SOC evolving between $25 \%$ and $85 \%$. Consequently, BESS protection and reliability are fully guaranteed.

Table 2 summarizes the performances of both controllers based IEMS, 3D-FL-FR, and ANN, with and without deloaded control. It shows the efficiency of the 3D-FL-FR based IEMS. After severe load variation, the frequency convergence is faster, and the frequency deviation is smaller, in comparison to the ANN-based controller.

\section{Conclusion}

A primary frequency regulation of a hybrid autonomous microgrid was proposed to improve the performances of autonomous microgrid. The WTGs, DG, and batteries were used as constituents to take advantage of their complementary features. To exploit the kinetic energy reserve, and regulate the frequency effectively and economically, the deloaded method of the WTG is adopted. Besides, the deloading is adjusted in a smart manner using 3D-FL-FR and ANN-based controllers. Consequently, the adopted approach allows relief for the DG reduces fuel consumption during the power unbalance, and increases the WTG output power. The results of the two proposed controllers have shown their benefits to assure the power balance and frequency stability even under severe load disturbances and large wind speed variation. Additionally, a combination of WTG deloaded mode and batteries, while using the adopted IEMS, enhances the A- $\mu \mathrm{G}$ energy efficiency, reduces the stress on the DG. The comparison study has also demonstrated the superiority of the 3D-FL-FR over ANN-based IEMS using MATLAB/Simulink environment. Moreover, the obtained results are in compliance with the IEEE Standards in terms of frequency deviation (Table 3).

The main findings of the present study can be summarized as follows:

- An IEMS is designed and proposed to improve the performance and the capabilities of an autonomous smart hybrid $\mu \mathrm{G}$.

Table 2 Results evaluation

\begin{tabular}{|c|c|c|c|c|c|c|c|c|}
\hline \multirow{3}{*}{$\begin{array}{l}\text { Load Variation } \\
\text { per time (s) }\end{array}$} & \multicolumn{4}{|c|}{ With 3D-FL-FR } & \multicolumn{4}{|c|}{ With ANN } \\
\hline & \multicolumn{2}{|l|}{$\Delta \mathrm{f}(\mathrm{Hz})$} & \multicolumn{2}{|l|}{$\Delta \mathrm{P}(\mathrm{MW})$} & \multicolumn{2}{|l|}{$\Delta \mathrm{f}(\mathrm{Hz})$} & \multicolumn{2}{|l|}{$\Delta \mathrm{P}(\mathrm{MW})$} \\
\hline & With DC & Without DC & With DC & Without DC & With DC & Without DC & With DC & Without DC \\
\hline 140 & -0.04 & -0.9 & +1 & 0 & -0.08 & -1.3 & +1.1 & 0 \\
\hline 270 & -1.4 & -6.3 & +2.8 & 0 & -1.8 & -6.7 & +2.7 & 0 \\
\hline 370 & +0.1 & -2.4 & -0.16 & 0 & +0.1 & -2.8 & -0.17 & 0 \\
\hline
\end{tabular}


Table 3 The system parameter

\begin{tabular}{lll}
\hline Symbol & Quantity & Value \\
\hline$P_{r}$ & rated power & $2 \mathrm{MW}$ \\
$P_{d}$ & deloaded power & $0.9 \mathrm{P}_{\max } \mathrm{MW}$ \\
$f$ & frequency & $50 \mathrm{~Hz}$ \\
$v$ & rated wind speed & $12.4 \mathrm{~m} / \mathrm{s}$ \\
$S$ & swept area & $4775.94 \mathrm{~m}^{2}$ \\
$\omega_{m-r e f}$ & Rated Rotational Speed & $2.57 \mathrm{rad} / \mathrm{s}$ \\
$R_{i}$ & internal resistance & $80 \mathrm{~m} \Omega$ \\
$n_{b}$ & cells number in series & 72 \\
$I_{n}$ & rated current & $120 \mathrm{~A}$ \\
$k_{G}$ & Generator gain & 1 \\
$\tau_{G}$ & Generator time constant & $0.1 \mathrm{~s}$ \\
$k_{e}$ & DG factor & 7 \\
$k_{e}$ & Engine gain & 1 \\
$\tau_{e}$ & Engine time constant & $0.25 \mathrm{~s}$ \\
\hline
\end{tabular}

- Combination of WTG deloaded mode and batteries, using the adopted IEMS, enhances the A- $\mu \mathrm{G}$ energy efficiency, reduces the stress on the DG, extracts the maximum power from renewables, and minimizes the current fluctuation in the BESS;

- Both intelligent based controllers guarantee a frequency deviation that meets the IEEE Std 1547-2003:

- With DC and 3D-FL-FR, the frequency deviation is kept at $-2.8 \%$ during $2 \mathrm{~s}$;

- The combination of DC and ANN exhibits a frequency drop of $-3.6 \%$, during $3 \mathrm{~s}$.

\section{References}

1. Zhang S, Mishra Y, Shahidehpour M (2016) Fuzzy-Logic Based Frequency Controller for Wind Farms Augmented With Energy Storage Systems. IEEE Trans Power Syst 31(2):1595-1603

2. Bukar AL, Tan CW, Lau KY (2019) Optimal sizing of an autonomous photovoltaic/wind/battery/diesel generator microgrid using grasshopper optimization algorithm. Sol Energy 188:685-696

3. Al Busaidi AS, Kazem HA, Al-Badi AH, Farooq Khan M (2016) A review of optimum sizing of hybrid PV-Wind renewable energy systems in oman. Renew Sust Energ Rev 53:185-193

4. Djellouli A, Lakdja F, Rachid M (2019) "Control and Management of Hybrid Renewable Energy System," in Proceedings of the 1 st International Conference on Smart Innovation, Ergonomics and Applied Human Factors (SEAHF), p. 1-10

5. Li Y, Yang Z, Li G, Zhao D, Tian W (2019) Optimal Scheduling of an Isolated Microgrid With Battery Storage Considering Load and Renewable Generation Uncertainties. IEEE Trans Ind Electron 66(2):1565-1575

6. Pathak G, Singh B, Panigrahi BK (2016) Control of Wind-Diesel Microgrid Using Affine Projection-Like Algorithm. IEEE Trans Ind Inform 12(2):524-531
7. Dufo-López R, Cristóbal-Monreal IR, Yusta JM (2016) Stochastic-heuristic methodology for the optimisation of components and control variables of PV-wind-diesel-battery stand-alone systems. Renew Energy 99:919-935

8. Shivaiea M, Mokhayeric M, Kiani-Moghaddam M, AshouriZadeh A (2019) A reliability-constrained cost-effective model for optimal sizing of an autonomous hybrid solar/wind/diesel/ battery energy system by a modified discrete bat search algorithm. Solar Energy

9. Moghaddam IN, Chowdhury BH, Doostan M (2018) Optimal sizing and operation of battery energy storage systems connected to wind farms participating in electricity markets. IEEE Trans Sustain Energy:1-1

10. Yang Y, Bremner S, Menictas C, Kay M (2018) Battery energy storage system size determination in renewable energy systems: A review. Renew Sust Energ Rev 91:109-125

11. Wang C, Li J, Hu Y (2019) Frequency control of isolated winddiesel microgrid power system by double equivalent input-disturbance controllers. IEEE Access

12. Chauhan A, Saini RP (2014) A review on Integrated Renewable Energy System based power generation for stand-alone applications: Configurations, storage options, sizing methodologies and control. Renew Sust Energ Rev 38:99-120

13. Zou C, Zhang L, Hu X, Wang Z, Wik T, Pecht M (2018) A review of fractional-order techniques applied to lithium-ion batteries, lead-acid batteries, and supercapacitors. J Power Sources 390:286-296

14. Feng X, Gu J, Guan X (2018) Optimal allocation of hybrid energy storage for microgrids based on multi-attribute utility theory. J Mod Power Syst Clean Energy 6(1):107-117

15. Díaz-González F, Sumper A, Gomis-Bellmunt O, Villafáfila-Robles R (2012) A review of energy storage technologies for wind power applications. Renew Sust Energ Rev 16(4):2154-2171

16. Chandra S, Agrawal S, Chauhan DS (2018) Soft computing based approach to evaluate the performance of solar PV module considering wind effect in laboratory condition. Energy Rep 4:252-259

17. Bouchebbat R, Gherbi S (2017) A Novel Optimal Control and Management Strategy of Stand-Alone Hybrid PV/Wind/Diesel Power System. J Control Autom Electr Syst 28(2):284-296

18. Dettori S, Iannino V, Colla V, Signorini A (2018) An adaptive Fuzzy logic-based approach to PID control of steam turbines in solar applications. Appl Energy 227:655-664

19. Pelletier F, Masson C, Tahan A (2016) Wind turbine power curve modelling using artificial neural network. Renew Energy 89:207-214

20. Mahrouch A, Ouassaid M (2019) "Primary Frequency Control for Wind/Battery Stand-Alone Microgrid," 2019 7th International Renewable and Sustainable Energy Conference (IRSEC), Agadir, Morocco, pp. 1-6

21. Mahrouch A, Ouassaid M (2020) "Primary Frequency Regulation of Isolated Microgrid based on Three-Dimensional Fuzzy Logic Control for Deloaded PMSG Enhanced by Diesel Generator," 2020 International Conference on Electrical and Information Technologies (ICEIT), Rabat, Morocco, pp. 1-6

22. Li J et al (2020) Optimal reactive power dispatch of permanent magnet synchronous generator-based wind farm considering levelised production cost minimisation. Renew Energy 145:1-12

23. Chub A, Husev O, Blinov A, Vinnikov D (2017) Novel Isolated Power Conditioning Unit for Micro Wind Turbine Applications. IEEE Trans Ind Electron 64(7):5984-5993

24. Mendis N, Muttaqi KM, Perera S (2014) Management of BatterySupercapacitor Hybrid Energy Storage and Synchronous Condenser for Isolated Operation of PMSG Based Variable-Speed Wind Turbine Generating Systems. IEEE Trans Smart Grid 5(2):944-953 
25. Tu T, Rajarathnam GP, Vassallo AM (2019) Optimization of a stand-alone photovoltaic-wind-diesel-battery system with multilayered demand scheduling. Renew Energy 131:333-347

26. Mir AS, Senroy N (2020) Self-Tuning Neural Predictive Control Scheme for Ultrabattery to Emulate a Virtual Synchronous Machine in Autonomous Power Systems. IEEE Trans Neural Netw Learn Syst 31(1):136-147

27. Faisal M, Hannan MA, Ker PJ, Uddin MN (2019) Backtracking search algorithm based fuzzy charging-discharging controller for battery storage system in microgrid applications. IEEE Access 7:159357-159368

28. Marzebali MH, Mazidi M, Mohiti M (2020) An adaptive droopbased control strategy for fuel cell-battery hybrid energy storage system to support primary frequency in stand-alone microgrids. J Energy Storage 27:101127

29. El-Bidairi KS, Nguyen HD, Mahmoud TS, Jayasinghe SDG, Guerrero JM (2020) Optimal sizing of Battery Energy Storage Systems for dynamic frequency control in an islanded microgrid: A case study of Flinders Island, Australia. Energy 195:117059

30. Akram U, Nadarajah M, Shah R, Milano F (2020) A review on rapid responsive energy storage technologies for frequency regulation in modern power systems. Renew Sust Energ Rev 120:109626

31. Rehman S, Habib HUR, Wang S, Büker MS, Alhems LM, Al Garni HZ (2020) Optimal design and model predictive control of standalone HRES: a real case study for residential demand side management. IEEE Access 8:29767-29814

32. Wang C, Li J, Hu Y (2019) Frequency control of isolated winddiesel microgrid power system by double equivalent-input-disturbance controllers. IEEE Access 7:105617-105626

33. Pradhan C, Bhende CN (2015) Adaptive deloading of standalone wind farm for primary frequency control. Energy Syst 6(1):109-127

34. Vidyanandan KV, Senroy N (2013) Primary frequency regulation by deloaded wind turbines using variable droop. IEEE Trans Power Syst 28(2):837-846
35. Wang C, Liu Y, Li X, Guo L, Qiao L, Lu H (2016) Energy management system for stand-alone diesel-wind-biomass microgrid with energy storage system. Energy 97:90-104

36. Ogunjuyigbe ASO, Ayodele TR, Akinola OA (2016) Optimal allocation and sizing of PV/Wind/Split-diesel/Battery hybrid energy system for minimizing life cycle cost, carbon emission and dump energy of remote residential building. Appl Energy 171:153-171

37. Wang H, Yang J, Chen Z, Ge W, Ma Y, Xing Z (2018) Model Predictive Control of PMSG-Based Wind Turbines for Frequency Regulation in an Isolated Grid. IEEE Trans Ind Appl 54:3077-3089

38. Paul Ayeng'o S, Schirmer T, Kairies K-P, Axelsen H, Uwe Sauer D (2018) Comparison of off-grid power supply systems using lead-acid and lithium-ion batteries. Sol Energy 162:140-152

39. Achaibou N, Haddadi M, Malek A (2012) Modeling of Lead Acid Batteries in PV Systems. Energy Procedia 18:538-544

40. Elyaalaoui K, Ouassaid M, Cherkaoui M (2018) Primary frequency control using hierarchal fuzzy logic for a wind farm based on SCIG connected to electrical network. Sustain Energy Grids Netw 16:188-195

41. Yilmaz AS, Özer Z (2009) Pitch angle control in wind turbines above the rated wind speed by multi-layer perceptron and radial basis function neural networks. Expert Syst Appl 36(6):9767-9775

42. "IEEE Standard for Interconnecting Distributed Resources with Electric Power Systems," in IEEE Std 1547-2003, vol., no., pp.1-28, 2003

Publisher's Note Springer Nature remains neutral with regard to jurisdictional claims in published maps and institutional affiliations. 